\title{
The SWARM-BOTS Project
}

\author{
Marco Dorigo ${ }^{1}$, Elio Tuci ${ }^{1}$, Roderich Groß ${ }^{1}$, Vito Trianni ${ }^{1}$, \\ Thomas Halva Labella ${ }^{1}$, Shervin Nouyan ${ }^{1}$, Christos Ampatzis ${ }^{1}$, \\ Jean-Louis Deneubourg ${ }^{2}$, Gianluca Baldassarre ${ }^{3}$, Stefano Nolfi ${ }^{3}$, \\ Francesco Mondada $^{4}$, Dario Floreano ${ }^{4}$, and Luca Maria Gambardella ${ }^{5}$ \\ 1 IRIDIA, Université Libre de Bruxelles, Belgium \\ \{mdorigo, etuci,rgross, vtrianni, hlabella, snouyan, campatzi\}@ulb.ac.be \\ http://iridia.ulb.ac.be/ \\ 2 CENOLI, Université Libre de Bruxelles, Belgium \\ jldeneub@ulb.ac.be \\ http://www.ulb.ac.be/cenoliw3/ \\ 3 Institute of Cognitive Science and Technology, CNR, Rome, Italy \\ baldassarre@ip.rm.cnr.it, s.nolfi@istc.cnr.it \\ http://gral.ip.rm.cnr.it/ \\ 4 ASL, École Polytechnique Fédérale de Lausanne, Switzerland \\ \{francesco.mondada, dario.floreano\}@epfl.ch \\ http://lsa.epfl.ch/ \\ 5 IDSIA, Lugano, Switzerland \\ luca@idsia.ch \\ http://www.idsia.ch/
}

\begin{abstract}
This paper provides an overview of the SWARM-BOTS project, a robotic project sponsored by the Future and Emerging Technologies program of the European Commission. The paper illustrates the goals of the project, the robot prototype and the 3D simulator we built. It also reports on the results of experimental work in which distributed adaptive controllers are used to control a group of real, or simulated, robots so that they perform a variety of tasks which require cooperation and coordination.
\end{abstract}

\section{Vision}

This paper introduces and illustrates the theoretical underpinning and the research agenda of the SWARM-BOTS project, a robotic project sponsored by the Future and Emerging Technologies program of the European Commission (IST-2000-31010). The aim of this project is the development of a new robotic system, called a swarm-bot, based on swarm robotics techniques.

Swarm robotics is an emergent field of collective robotics that studies robotic systems composed of swarms of robots tightly interacting and cooperating to reach their goals [1]. Swarm robotics finds its theoretical roots in recent studies of animal societies, such as ants and bees. Social insects are a valuable source of inspiration for designing collectively intelligent systems comprising many agents. 
Despite noise in the environment, errors in processing information and performing tasks, and no global information, social insects are quite successful at performing group-level tasks. Based on the social insect metaphor, swarm robotics emphasises aspects such as decentralisation of the control, limited communication abilities among robots, use of local information, emergence of global behaviour and robustness [2].

The work carried out within the SWARM-BOTS project is directly inspired by the collective behaviour of social insects colonies and other animal societies, and in particular it focuses on the study of the mechanisms which govern the processes of self-organisation and self-assembling in artificial autonomous agents. In order to pursue these objectives, a new type of robot, referred to as $s$-bot, has been developed. Hardware development runs in parallel with the development of distributed adaptive architectures that make the $s$-bots capable of autonomously carrying out individual and collective behaviour by exploiting local interactions among the $s$-bots and between the $s$-bots and their environment.

The $s$-bots are mobile robots with the ability to connect to and disconnect from each other $[3,4]$. A swarm-bot is defined as an artifact composed of a swarm of assembled $s$-bots (see Figure 1 ). S-bots have relatively simple sensors and motors and limited computational capabilities. Their physical links are used to assemble into a swarm-bot able to solve problems that cannot be solved by a single $s$-bot. In the swarm-bot form, the s-bots are attached to each other as a single robotic system that can move and reconfigure. Physical connections between $s$-bots are essential for solving many collective tasks. For example, $s$-bots can form pulling chains to retrieve a heavy object (see Figure 1a). Also, during navigation on rough terrain, physical links can serve as support if the swarm-bot has to pass over a hole larger than a single $s$-bot (see Figure 1b), or when it has to pass through a steep concave region. However, for tasks such as searching for a goal location or tracing a path to a goal, a swarm of unconnected s-bots can be more efficient.

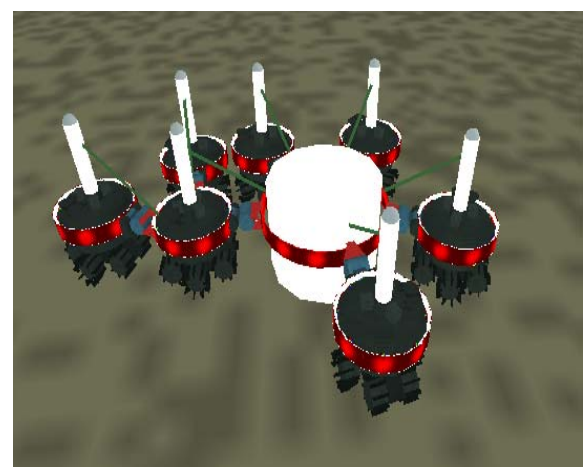

(a)

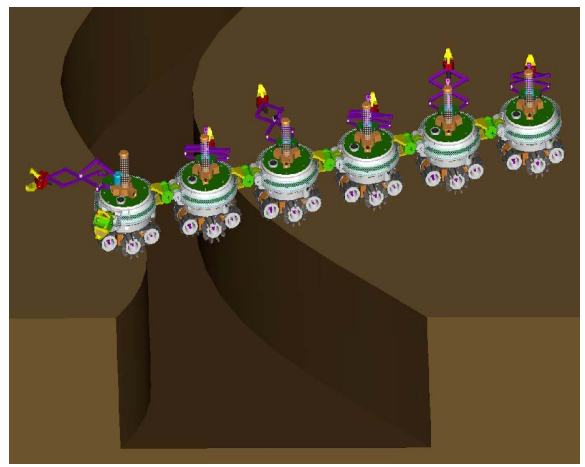

(b)

Fig. 1. Graphic visualisation of how the rigid gripper can be used to connect in a secure way $s$-bots among themselves so that they form a swarm-bot for (a) retrieving heavy objects or (b) passing over holes. 
The design and realisation of both the hardware and the software of such a robotic system represents the scientific challenge of the SWARM-BOTS project. In what follows, we first give a brief description of the robot hardware, and of the experimental methodology employed to develop the $s$-bots controllers (see Section 2). Then, in Section 3 we describe the results of several experiments in which controllers have been designed to allow the s-bots to autonomously perform a variety of individual and collective behaviours in partially or totally unknown environments. Discussion and conclusions can be found in Section 4.

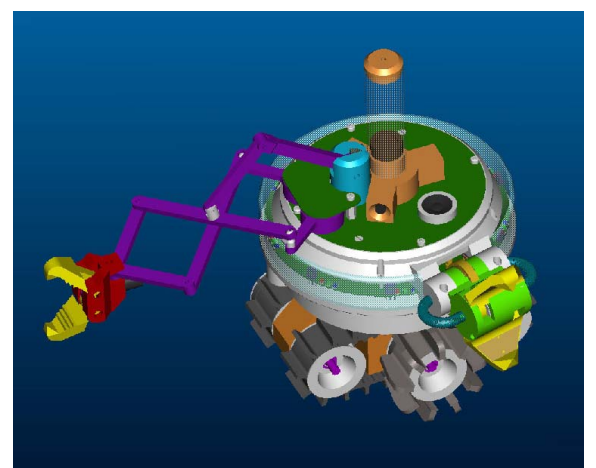

(a)

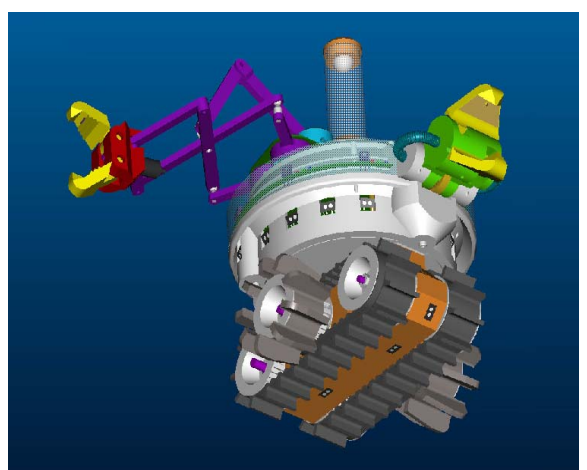

(b)

Fig. 2. Graphic visualisation of the s-bot concept. (a) The main body (turret), which has a diameter of $116 \mathrm{~mm}$, is equipped with passive and active gripping facilities, sensors and electronics. (b) The lower body (traction system) is equipped with tracks and hosts the batteries.

\section{The Hardware and the Simulation Environment}

The construction of a number of artifacts (30-35) capable of self-assembling and self-organising represents one of the most significant scientific challenges faced by the SWARM-BOTS project. In subsection 2.1, we briefly describe the hardware of the $s$-bots, with particular reference to its sensor and motor apparatus. A more detailed description of the hardware components can be found in [5]. In subsection 2.2, we briefly introduce the main features of swarmbot3d, a simulation environment employed to design the software which controls the $s$-bots ${ }^{1}$.

\subsection{The $s$-bot}

An $s$-bot is the basic elementary unit of the swarm-bot (see Figure 2). Each s-bot is a fully autonomous mobile robot capable of performing simple tasks such as autonomous navigation, perception of the environment and grasping of objects. In addition to these features, one $s$-bot is able to communicate with other $s$-bots

\footnotetext{
${ }^{1}$ Details regarding the hardware and simulation of the swarm-bot can also be found on the project web-site (www.swarm-bots.org).
} 


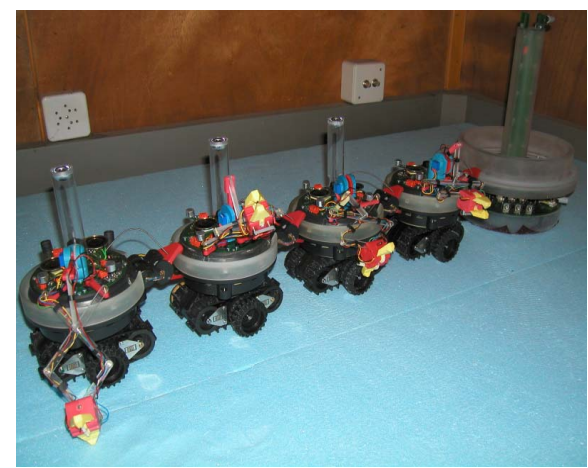

(a)

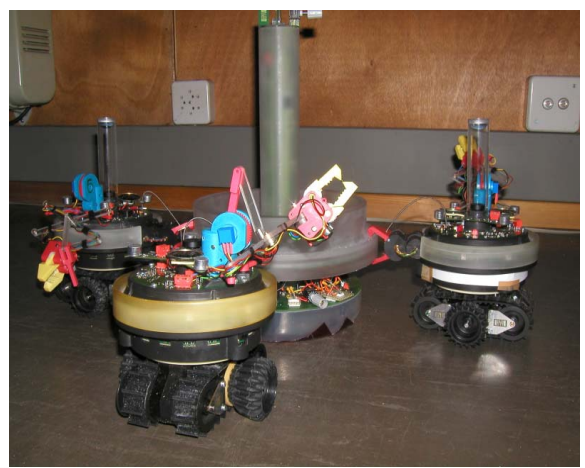

(b)

Fig. 3. Pictures of $s$-bots transporting an object that can not be moved by a single $s$ bot. (a) A swarm-bot comprising four s-bots pulls an object. (b) Three s-bots pull/push an object to which they are directly attached.

and physically connect to them, thus forming a so-called swarm-bot. A swarmbot is able to perform tasks in which a single $s$-bot has major problems, such as exploration, navigation, and transportation of heavy objects on rough terrain (see Figure 3).

As far as it concerns the mobility of the s-bot, an innovative system has been developed which makes use of both tracks and wheels as illustrated in Figure 2. The wheel and the track on a same side are driven by the same motor, building a differential drive system controlled by two motors. This combination of tracks and wheels is labelled Differential Treels ${ }^{\circledR}$ Drive $^{2}$. Such a combination has two advantages. First, it allows an efficient rotation on the spot due to the larger diameter and position of the wheels. Second, it gives to the traction system a shape close to the cylindrical one of the main body (turret), avoiding in this way the typical rectangular shape of simple tracks and thus improving the $s$-bot mobility.

The s-bot's traction system can rotate with respect to the main body by means of a motorised axis. Above the traction system, a rotating turret holds many sensory systems and the two grippers for making connections with other robots or objects. In particular, each s-bot is equipped with sensors necessary for navigation, such as infrared proximity sensors, light and humidity sensors, accelerometers and incremental encoders on each degree of freedom. Each robot is also equipped with sensors and communication devices to detect and communicate with other s-bots, such as an omni-directional camera, coloured LEDs around the robot's turret, and sound emitters and receivers. In addition to a large number of sensors for perceiving the environment, several sensors provide each $s$-bot with information about physical contacts, efforts, and reactions at the interconnection joints with other s-bots. These include torque sensors on most joints as well as traction sensors to measure the pulling/pushing forces exerted on the $s$-bot's turret.

\footnotetext{
${ }^{2}$ Treels is a contraction of TRacks and whEELS.
} 
$S$-bots have two types of possible physical interconnections for self-assembling into a swarm-bot configuration: rigid and semi-flexible. Rigid connections between two $s$-bots are established by a gripper mounted on a horizontal active axis (see Figure 2). Such a gripper has a very large acceptance area allowing it to realize a secure grasp at any angle and, if necessary, allowing it to lift another $s$-bot. Semi-flexible connections are implemented by a gripper positioned at the end of a flexible arm actuated by three servo-motors.

\subsection{The Simulation Environment: Swarmbot3d}

Swarmbot3d is a 3D dynamics simulator of our multi-agent system of cooperating robots, based on the SDK Vortex ${ }^{\mathrm{TM}}$ toolkit $^{3}$, which provides realistic simulations of dynamics and collisions of rigid bodies in 3D. Swarmbot3d provides s-bot models with the functionalities available on the real $s$-bots (see [5] for details). It can simulate different sensor devices such as IR proximity sensors, an omnidirectional camera, an inclinometer, sound, and light sensors.

A fundamental feature of the swarmbot3d simulator is that it provides robot simulation modules at different levels of detail. In particular, it provides a hierarchy of four $s$-bot reference models with increasing levels of detail. The less detailed models have been employed to speed up the process of designing neural controllers through evolutionary algorithms. The most detailed models have been employed to validate the evolved controllers before porting them on real hardware. The advantages of such a simulation environment are multiple: it works as an aiding tool for accurately predicting 3D kinematics and dynamics of a single $s$-bot in a swarm-bot; it has been employed to evaluate possible new options for hardware parts; it represents a "plastic" world model which allows the design of new experimental setups in 3D; it has been employed to quickly evaluate new distributed control ideas before porting them to the real hardware. Furthermore, the simulator provides on-line interactive control during simulation, useful for rapid prototyping of new control algorithms. Swarmbot3d allows to handle a group of robots either as independent units or in a swarm-bot configuration, which can be thought of as a graph, in which each node represents a connected s-bot. The connections can be created and released dynamically at simulation time. Connections may be of a rigid nature giving to the resulting structure the solidity of a whole entity.

\section{Results}

In this section, we briefly summarise the methods and the results of experimental work in which controllers have been designed to allow the $s$-bots to autonomously display a variety of individual and collective behaviours in partially and totally unknown environments. These basic behaviours represent different lines of investigation which are pursued in parallel, and are focused on: 1) aggregation; 2) coordinated motion; 3) collective and cooperative transport of a prey item;

\footnotetext{
${ }^{3}$ Critical Mass Labs, Canada (www.criticalmasslabs.com).
} 
4) exploration; 5) adaptive task allocation; 6) navigation on rough terrain; 7) functional self-assembling. These research lines have been identified by looking at the kind of requirements that either a single $s$-bot or an aggregation of $s$-bots must fulfil in order to successfully perform the tasks involved in a complex scenario. The latter requires a swarm of up to 35 s-bots to transport heavy objects from their initial location to a goal location in an environment which presents difficulties of various nature, such as obstacles and holes on the ground. Moreover, the weight and/or size of the objects to be transported are such that these objects can not be transported by a single $s$-bot (see Figure 4).

To be capable of accomplishing the scenario, the $s$-bots must be equipped with controllers that allow them to successfully navigate in a totally or partially unknown environment in order to find and retrieve a target. The s-bots must also be capable of aggregating and self-assembling in a swarm-bot formation. The swarm-bot might be of fundamental importance for passing over a hole larger than a single $s$-bot, or to retrieve objects that can not be transported by a single $s$-bot. Finally, a group of $s$-bots should be capable of adaptively allocating resources to different tasks to be carried out either sequentially or in parallel. For example, if two heavy objects must be transported, a group of $s$-bots must be capable of splitting into two sub-groups each of which is formed by the number of $s$-bots appropriately chosen with respect to the nature of the object to be transported. The following subsections illustrate the research activities concerning the development of the basic behavioural capabilities above mentioned.

\subsection{Aggregation}

Within the SWARM-BOTS project, aggregation is of particular interest since it stands as a prerequisite for other forms of cooperation. For instance, in order to assemble into a swarm-bot, s-bots should first be able to aggregate. Several experiments have focused on the design of scalable aggregation behaviours by means of sound signalling (see $[6,7]$ for details). Artificial neural networks shaped by evolutionary algorithms control the behaviour of a homogeneous group of $s$-bots (i.e., within a group, all the $s$-bots share the same controller). During the evolutionary phase, the groups are randomly placed in a square arena. The agents are equipped with a simulated speaker that can emit a tone for long range signalling. S-bots can perceive the intensity of sound using three sound sensors that simulate three directional microphones. The $s$-bot controller takes as input the state of the $s$-bot proximity sensors, and the state of the sound sensors. Two output nodes control the $s$-bot's motors. Controllers that exploit sound to let a group of $s$-bots aggregate are evolved using a fitness function that selectively rewards those groups which minimise the average distance of all the $s$-bots from the group centre of mass.

The evolved controllers show quite robust aggregation strategies. In particular, the s-bots exploit the sound signal both to get closer to each other, and to remain aggregated. In general, all evolved strategies rely on a delicate balance between attraction to sound sources and repulsion from other robots, the former 


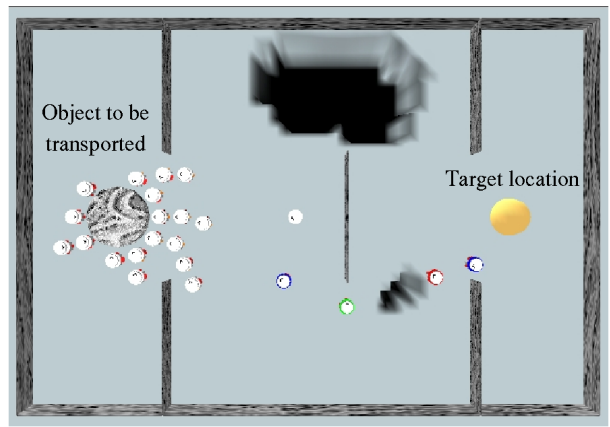

Fig. 4. The scenario: a swarm of up to 35 s-bots must transport a heavy object from an initial to a goal location. The cylinder on the left side represents the object to be transported; the landmark on the right side represents the target location where the object has to be transported. The four $s$-bots between the cylindrical object and the target location form a path which logically connects the former to the latter. This path is exploited by other s-bots to move back and forth between the target location and the object to be retrieved. Also visible are two types of obstacles: walls and holes.

being perceived by sound sensors, the latter by proximity sensors. A qualitative analysis of the evolved controllers reveals that different replications result in slightly different behaviours. In particular, the evolved solutions differ mainly in the behaviour of $s$-bots when they are close to each other.

Further evaluation tests concerning scalability of the evolved solutions have shown that controllers evolved for groups of four $s$-bots can successfully bring forth aggregation in groups with a higher number of $s$-bots (up to 40 s-bots). The best scalable strategy was the one in which the controller creates an aggregate that moves across the arena. This is a result of the complex motion of $s$-bots within the aggregate, which in turn is the result of the interaction between attraction to sound sources and repulsion from other robots. The slow motion of the aggregate across the arena leads to scalability, as an aggregate can continue to move joining solitary s-bots or other already formed aggregates, eventually forming a single cluster of $s$-bots.

\subsection{Coordinated Motion}

Coordinated motion represents another basic ability for a swarm-bot formed of connected $s$-bots that, being independent of each other in their control, must coordinate their actions to choose a common direction of motion. The coordinated motion ability is essential for an efficient motion of the swarm-bot as a whole, and it is achieved mainly through the exploitation of the information coming from the traction sensor, which is placed at the turret-chassis junction of an $s$-bot. The traction sensor returns the direction (i.e., the angle with respect to the chassis' orientation) and the intensity of the force of traction (henceforth called "traction") that the turret exerts on the chassis. Traction is caused by the movement of both the connected $s$-bots and the $s$-bot's chassis. Note that the 
turret of each $s$-bot physically integrates the forces that are applied to the $s$-bot by the other $s$-bots. As a consequence, the traction sensor provides the $s$-bot with an indication of the average direction toward which the group is trying to move as a whole. More precisely, it measures the mismatch between the directions toward which the entire group and the s-bot's chassis are trying to move. The intensity of traction measures the size of this mismatch.

Our experimental work has focused on the evolution of artificial neural networks capable of coordinately controlling the behaviour of a swarm-bot (a collection of assembled $s$-bots). In this kind of experiments, the problem that the $s$-bots have to solve is that their wheels might have different initial directions or might mismatch while moving. In order to coordinate, s-bots should be able to collectively choose a common direction of movement having access only to local information (see Figure 5). Each s-bot's controller (i.e., an artificial neural network), takes as input the reading of its traction sensor and other sensor readings, and sets the status of the $s$-bot' actuators.

The results show that evolution can find simple and effective solutions that allow the $s$-bots to move in a coordinate way independently of the topology of the swarm-bot and of the type of link with which the $s$-bots are connected (semi-flexible or rigid). Moreover, it is shown that the evolved $s$-bots also exhibit obstacle avoidance behaviour (when placed in an environment with obstacles) and object pulling/pushing behaviour (when assembled to or around an object, see Figure 6), and scale well to swarm-bots of a larger size (see [8,9] for details).

\subsection{Collective and Cooperative Transport of a Prey Item}

By taking inspiration from the behaviour of ants, the SWARM-BOTS project aims to build autonomous agents which by solely relying on local information, are capable of cooperatively and collectively carrying objects which can not be

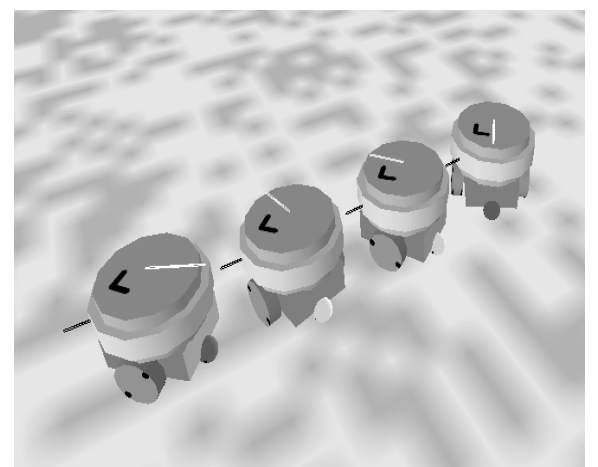

(a)

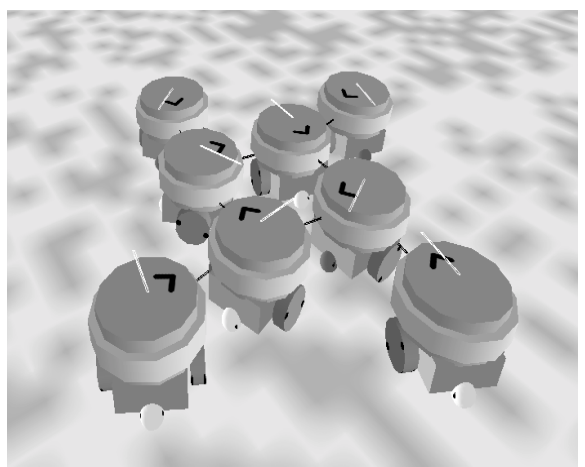

(b)

Fig. 5. (a) Four physically linked s-bots forming a linear structure. The lines between two $s$-bots represent the physical link between them. The white line above each $s$-bot indicates the direction and intensity of the traction. (b) Eight $s$-bots connected by rigid links into a "star formation". 
moved by a single agent. The members of a group have to coordinate their actions to achieve the desired outcome. In particular, due to the nature of the object (i.e., its shape, dimension, and weight) the $s$-bots might be required to connect to each other in swarm-bot formation and/or to the object itself for transporting it (i.e., gripping the object with the fixed gripper, see Figure 7).

In a series of experimental works, artificial neural networks have been evolved to control the actions of a single homogeneous group of $s$-bots which is required to pull and/or push an object in an arbitrarily chosen direction. During the evolutionary phase, the $s$-bots are located in a boundless arena, in the proximity of objects of various shape, dimension, and weight. Only indirect communication through the environment can be exploited to attain coordination. The evolved controllers exhibit rather good transport performances. Certain controllers show scaling properties: they can be applied to larger groups of s-bots to move bigger and heavier prey objects. However, the controllers' performances are very sensitive to the size of the prey (see [10]).

A follow-up work focused on the self-organisation of $s$-bots into assembled structures and on the transport of heavy prey by groups of assembled $s$-bots to a target. To facilitate the process of assembling, the $s$-bots are provided with the ability to detect teammates; in addition, the presence of assembled structures is favoured by the fitness function employed. The best evolved controller proved fairly robust with respect to different combinations of size and shape of the prey (see [11]).

Recently, the situation has been studied in which some $s$-bots are given the opportunity to localise the transport target, while the others (called the blind ones) are not. To enable a blind s-bot to contribute to the group's performance, it has been equipped with sensors to perceive both whether or not it is moving, and traction forces on its turret. For group sizes ranging from 2 to 16, it has been shown that blind s-bots make an essential contribution to the group's per-

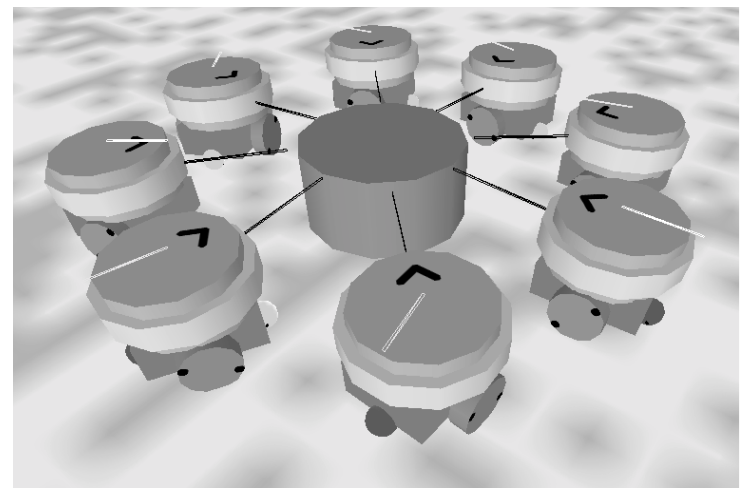

(a)

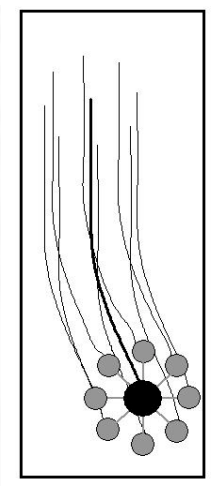

(b)

Fig. 6. (a) Eight $s$-bots connected to an object through rigid links. (b) Traces left by the $s$-bots (thin lines) and the object (thick line) during 150 simulation cycles. The gray and black circles represent the initial positions of the $s$-bots and of the object. 


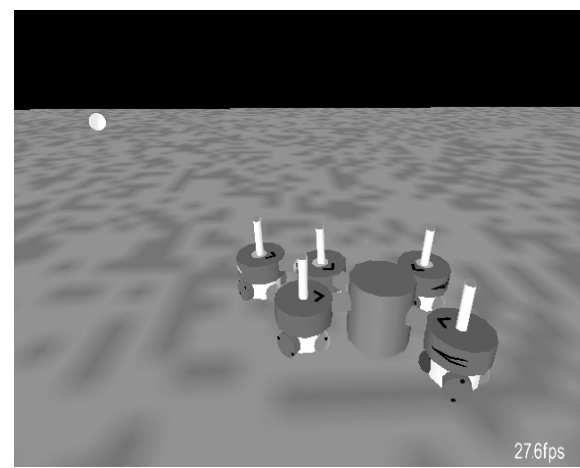

(a)

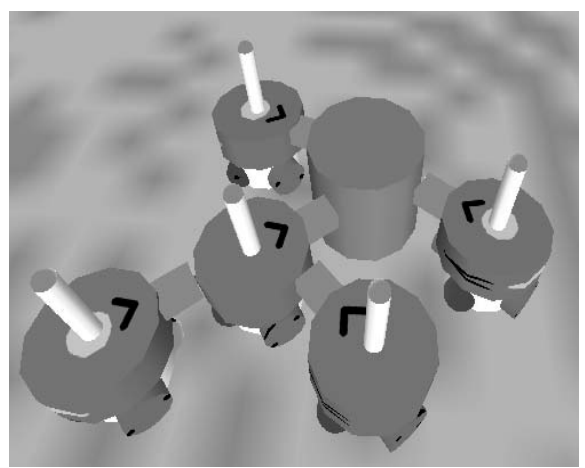

(b)

Fig. 7. (a) S-bots connected to each other and to an object. (b) A closer view on the connections.

formance. For the best evolved solution the performance scales well with group size, making possible the transport of heavier prey by larger swarms of blind and non-blind s-bots (see [12]).

\subsection{Exploration}

This subsection illustrates the mechanisms employed by the $s$-bots to efficiently explore a partially or totally unknown environment. Our approach is based on the exploitation of the collectivity, and it requires that some s-bots - referred to as $s$-bot beacons - be capable of positioning themselves in the environment in order to work as beacons for other s-bots - referred to as s-bot explorers that move back and forth from a starting position to a goal location. The $s$-bot beacons should form a chain which connects different locations that cannot be perceived simultaneously by a single s-bot. In this way, a path between a goal and a home location is established, and it can be subsequently exploited by the $s$-bot explorers. The main advantage of this exploration strategy is that it does not require the $s$-bots to create a map-like representation of the world.

The status of these experiments, in which a behaviour-based approach is employed to design the $s$-bots controllers, is still preliminary. However, simply by varying two parameters of the s-bots controller (i.e., the probability of each single agent to become a beacon and the probability of a robot beacon to become an explorer) it is possible to bring forth a variety of exploration strategies each of which results more adaptive in certain types of environment than in others. Up to date, two different strategies have been implemented. In the simplest setup, we have static chains: the $s$-bots beacons do not move. In the other setup, the s-bots that form a chain move coordinately without breaking the chain. We are currently working on the development of an adaptive mechanism which autonomously sets these parameters with respect to the characteristics of the environment experienced by the s-bots. 


\subsection{Adaptive Task Allocation}

Task allocation and division of labour are two important research areas within collective robotics. Previous studies have shown that small groups of robots might perform a collective task similar or better than a larger group. However, this efficiency loss can be avoided if large groups of robots are equipped with an adaptive task allocation mechanism which distributes the resources of the group with respect to the nature of the task and the diversity among the individuals of the group. Within the SWARM-BOTS project we are obviously interested in designing an adaptive task allocation mechanism which allocates to each task a sufficient number of s-bots without reducing the efficiency of the entire group. In particular, we have been working on a mechanism which adaptively tunes the number of active agents in a foraging task: that is, searching for objects and retrieving them to a nest location. The agents, controlled by a behaviour-based architecture, use a simple adaptive mechanism which adjusts the probability of each agent to be a forager with respect to the current success rate of the individual on the task. Owing to this simple adaptive mechanism, a self-organised task allocation is observed at the global level. That is, not all the agents end up being active foragers. The same mechanism is also effective in exploiting mechanical differences among the robots inducing specialisation in the robots' activities. More details are given in $[13,14]$.

\subsection{Navigation on Rough Terrain}

Navigating on rough terrain is an important feature for an adaptive autonomous system. It can apply to many possible application scenarios, like space exploration or rescue in a collapsed building. Within the SWARM-BOTS project, several experiments have been run on an instance of the family of navigation on rough terrain tasks, that is, hole avoidance. A swarm-bot is required to perform coordinated motion in an environment that presents holes too large to be traversed. Thus, holes must be recognised and avoided, so that the swarm-bot does not fall into them. The difficulty of this task is twofold: first, $s$-bots should coordinate their motion. Second, s-bots have to recognise the presence of a hole, communicate it to the whole group and re-organise to choose a safer direction of motion. The results demonstrate that the evolved controllers (i.e., artificial neural networks) manage to efficiently manoeuvre a swarm-bot in the proximity of holes in the ground. Evolution is able to produce a self-organising system that relies on simple and general rules, a system that is consequently robust to environmental changes and to the number of s-bots involved in the experiment. The evolved strategies strongly rely on the traction forces produced by those $s$-bots that feel the presence of a hazard (see [15] for details).

\subsection{Functional Self-assembling}

These studies focus on the design of controllers for a group of s-bots required to connect to each other, each time environmental contingencies prevent a sin- 
gle $s$-bot to achieve its goal [16]. We refer to this capability as functional selfassembling, since the self-organised creation of a physically connected structure has to be functional to the accomplishment of a particular task.

The complexity of functional self-assembling resides in the nature of the individual mechanisms required to bring forth the coordinated movements that lead firstly to the formation of the assembled structure, and subsequently to the collective motion of the assembled structure.

In a preliminary set of studies, we have focused on the evolution of neural controllers for self-assembling s-bots required to solve a simple scenario. In particular, we have investigated a scenario which requires the $s$-bots to approach a light source located at the end of a corridor. Assembling is required to navigate in a "low temperature" area in which a swarm-bot can navigate more effectively than a group of disconnected s-bots. When located in the low temperature area, the aggregation of the $s$-bots should facilitate the subsequent assembling through their gripper element. This experimental setup allows us to investigate the basic mechanisms that underpin functional self-assembling.

The results of our empirical work show that integrated (i.e., not modularised) artificial neural networks can be successfully synthesised by evolutionary algorithms in order to allow a group of s-bots to display individual and collective obstacle avoidance, individual and collective phototaxis, aggregation and selfassembling. To the best of our knowledge, our experiments represent one of the first works in which (i) functional self-assembling in a homogeneous group of robots has been achieved and (ii) evolved neural controllers successfully cope with a complex scenario, producing different individual and collective responses based on the appropriate control of the state of various actuators triggered by the local information coming from various sensors.

\section{Discussion and Conclusions}

In this paper we have illustrated the most important features of a novel robotic concept, called swarm-bot. A swarm-bot is a self-organising, self-assembling artifact composed of a variable number of autonomous elementary units, called $s$-bots. As illustrated in Section 2, each s-bot is a fully autonomous agent capable of displacement, sensing and acting based on local information. Moreover, the self-assembling ability of the $s$-bots enables a group of agents to execute tasks that are beyond the capabilities of the single robot.

Concerning the hardware, the presence of many of such autonomous entities that can self-assemble in a single body and disband any time the union is no longer required, makes the system extremely versatile and robust to failures. Contrary to the swarm-bot, other robotic systems composed of small elementary units capable of reconfiguring themselves are less versatile and less robust, due to the fact that each unit has no or very limited mobility, very limited sensing capabilities, and acts often under the control of a central unit (see [17-19]).

Concerning the s-bots' controllers, we have developed them making an extensive use of artificial neural networks shaped by evolutionary algorithms. The solutions found by evolution are simple and in many cases they generalise to 
different environmental situations. This demonstrates that evolution is able to produce a self-organised system that relies on simple and general rules, a system that is consequently robust to environmental changes and that scales well with the number of $s$-bots involved in the experiments.

\section{Acknowledgement}

This work was supported by the "SWARM-BOTS" project, funded by the Future and Emerging Technologies programme (IST-FET) of the European Commission, under grant IST-2000-31010. The information provided is the sole responsibility of the authors and does not reflect the Community's opinion. The Community is not responsible for any use that might be made of data appearing in this publication. Marco Dorigo acknowledges support from the Belgian FNRS, of which he is a Research Director, through the grant "Virtual Swarm-bots", contract no. 9.4515.03, and from the "ANTS" project, an "Action de Recherche Concertée" funded by the Scientific Research Directorate of the French Community of Belgium.

\section{References}

1. Dorigo, M., Şahin, E.: Swarm robotics - special issue editorial. Autonomous Robots 17 (2004) 111-113

2. Bonabeau, E., Dorigo, M., Theraulaz, G.: Swarm Intelligence: From Natural to Artificial Systems. Oxford University Press, New York, NY (1999)

3. Şahin, E., Labella, T.H., Trianni, V., Deneubourg, J.L., Rasse, P., Floreano, D., Gambardella, L.M., Mondada, F., Nolfi, S., Dorigo, M.: SWARM-BOT: Pattern formation in a swarm of self-assembling mobile robots. In: Proceedings of the IEEE International Conference on Systems, Man and Cybernetics, IEEE Press, Piscataway, NJ (2002)

4. Mondada, F., Pettinaro, G.C., Kwee, I.W., Guignard, A., Gambardella, L.M., Floreano, D., Nolfi, S., Deneubourg, J.L., Dorigo, M.: SWARM-BOT: A swarm of autonomous mobile robots with self-assembling capabilities. In Hemelrijk, C., Bonabeau, E., eds.: Proceedings of the International Workshop on Self-organisation and Evolution of Social Behaviour, Monte Verità, Ascona, Switzerland (2002) 307312

5. Mondada, F., Pettinaro, G.C., Guignard, A., Kwee, I.V., Floreano, D., Deneubourg, J.L., Nolfi, S., Gambardella, L.M., Dorigo, M.: SWARM-BOT: A new distributed robotic concept. Autonomous Robots 17 (2004) 193-221

6. Baldassarre, G., Nolfi, S., Parisi, D.: Evolving mobile robots able to display collective behaviour. Artificial Life 9 (2003) 255-267

7. Trianni, V., Groß, R., Labella, T.H., Şahin, E., Dorigo, M.: Evolving aggregation behaviors in a swarm of robots. In Banzhaf, W., Christaller, T., Dittrich, P., Kim, J.T., Ziegler, J., eds.: Proceedings of the Seventh European Conference on Artificial Life. Volume 2801 of Lecture Notes in Artificial Intelligence, Springer Verlag, Berlin, Germany (2003) 865-874

8. Baldassarre, G., Nolfi, S., Parisi, D.: Evolution of collective behaviour in a team of physically linked robots. In Günther, R., Guillot, A., Meyer, J.A., eds.: Proceedings of the Second European Workshop on Evolutionary Robotics (EvoWorkshops2003: EvoROB). Volume 2611 of Lecture Notes in Computer Science, Springer Verlag, Berlin, Germany (2003) 581-592 
9. Dorigo, M., Trianni, V., Şahin, E., Groß, R., Labella, T.H., Baldassarre, G., Nolfi, S., Deneubourg, J.L., Mondada, F., Floreano, D., Gambardella, L.M.: Evolving self-organizing behaviors for a swarm-bot. Autonomous Robots 17 (2004) 223-245

10. Groß, R., Dorigo, M.: Evolving a cooperative transport behavior for two simple robots. In Liardet, P., Collet, P., Fonlupt, C., Lutton, E., Schoenauer, M., eds.: Artificial Evolution - 6th International Conference, Evolution Artificielle. Volume 2936 of Lecture Notes in Computer Science., Springer Verlag, Berlin, Germany (2004) 305-317

11. Groß, R., Dorigo, M.: Cooperative transport of objects of different shapes and sizes. In Dorigo, M., Birattari, M., Blum, C., Gambardella, L.M., Mondada, F., Stützle, T., eds.: Proceedings of ANTS 2004 - Fourth International Workshop on Ant Colony Optimization and Swarm Intelligence. Volume 3172 of Lecture Notes in Computer Science., Springer Verlag, Berlin, Germany (2004) 107-118

12. Groß, R., Dorigo, M.: Group transport of an object to a target that only some group members may sense. In X. Yao et al., eds.: Proceedings of PPSN-VIII, Eighth International Conference on Parallel Problem Solving from Nature. Volume 3242 of Lecture Notes in Computer Science., Springer Verlag, Berlin, Germany (2004) $852-861$

13. Labella, T., Dorigo, M., Deneubourg, J.L.: Efficiency and task allocation in prey retrieval. In Ijspeert, A., Murata, M., Wakamiya, N., eds.: Proceedings of the First International Workshop on Biologically Inspired Approaches to Advanced Information Technology (Bio-ADIT2004). Volume 3141 of Lecture Notes in Computer Science., Springer Verlag, Heidelberg, Germany (2004) 32-47

14. Labella, T., Dorigo, M., Deneubourg, J.L.: Self-organised task allocation in a swarm of robots. Technical Report TR/IRIDIA/2004-6, Université Libre de Bruxelles, Belgium (2004) To appear in the 7th International Symposium on Distributed Autonomous Robotic Systems (DARS04), June 23-25, 2004, Toulouse, France.

15. Trianni, V., Nolfi, S., Dorigo, M.: Hole avoidance: Experiments in coordinated motion on rough terrain. In Groen, F., Amato, N., Bonarini, A., Yoshida, E., Kröse, B., eds.: Intelligent Autonomous Systems 8, IOS Press, Amsterdam, The Netherlands (2004) 29-36

16. Trianni, V., Tuci, E., Dorigo, M.: Evolving functional self-assembling in a swarm of autonomous robots. In Schaal, S., Ijspeert, A., Billard, A., Vijayakamur, S., Hallam, J., Meyer, J.A., eds.: From Animals to Animats 8. Proceedings of the Eight International Conference on Simulation of Adaptive Behavior (SAB04), MIT Press, Cambridge, MA (2004) 405-414

17. Yim, M., Duff, D.G., Roufas, K.D.: PolyBot: a modular reconfigurable robot. In: Proceedings of the 2000 IEEE International Conference on Robotics and Automation (ICRA 2000). Volume 1, IEEE Press, Piscataway, NJ (2000) 514-520

18. Castano, A., Shen, W.M., Will, P.: CONRO: Towards deployable robots with inter-robot metamorphic capabilities. Autonomous Robots 8 (2000) 309-324

19. Khosla, P., Brown, B., Paredis, C., Grabowski, B., Navarro, L., Bererton, C., Vandeweghe, M.: Millibot Report. Report on millibot project, DARPA contract DABT63-97-1-0003, Carnegie Mellon University, Pittsburgh, Pennsylvania, USA (2002) 